\title{
Stimulation of Nitric Oxide-cGMP Pathway Excites Striatal Cholinergic Interneurons via Protein Kinase G Activation
}

\author{
Diego Centonze, ${ }^{1,2}$ Antonio Pisani, ${ }^{1,2}$ Paola Bonsi, ${ }^{2}$ Patrizia Giacomini, ${ }^{3}$ Giorgio Bernardi,, ${ }^{1,2}$ and \\ Paolo Calabresi ${ }^{1,2}$
}

${ }^{1}$ Clinica Neurologica, Dipartimento di Neuroscienze, Università "Tor Vergata," 00133 Rome, Italy, 2/stituto di Ricovero e Cura a Carattere Scientifico, Fondazione Santa Lucia, 00179 Rome, Italy, and 3/l Clinica Neurologica, Università "La Sapienza," 00185 Rome, Italy

\begin{abstract}
Conflicting data have been collected so far on the action of nitric oxide (NO) on cholinergic interneurons of the striatum. In the present in vitro electrophysiological study, we reported that intracellularly recorded striatal cholinergic interneurons are excited by both hydroxylamine and $S$-nitroso- $N$-acetylpenicillamine, two NO donors. This excitation persisted unchanged in the presence of glutamate, dopamine, and substance $P$ receptor antagonists as well as after blockade of tetrodotoxin (TTX)and calcium channel-sensitive transmitter release, suggesting that NO produces its effects by modulating directly resting ion conductances in the somatodendritic region of striatal cholinergic cells. The depolarizing effect of hydroxylamine was greatly reduced by lowering external concentrations of sodium ions (from 126 to $38 \mathrm{~mm}$ ) and did not reverse polarity in the voltage range from -120 to $-40 \mathrm{mV}$. The sodium transporter blockers bepridil and $3^{\prime}, 4^{\prime}$-dichlorobenzamil were conversely ineffective in preventing $\mathrm{NO}$-induced membrane depolarization. Intracellular cGMP elevation is required for the action of hydroxylamine
\end{abstract}

In recent years, electrophysiological, histochemical, and morphological criteria allowed the identification of distinct classes of striatal interneurons. They comprise $\sim 2-4 \%$ of the neuronal population of the striatum, the remaining being represented by medium spiny GABAergic projection cells. According to their physiological characteristics, striatal interneurons have been divided into fast-spiking (FS), low-threshold spikes (LTS), and long-lasting afterhyperpolarization (LA) cells (Kawaguchi, 1993; Kawaguchi et al., 1995). FS and LTS cells are GABAergic interneurons and are immunoreactive for parvalbumin and nitric oxide synthase (NOS), respectively. LA cells, conversely, the most studied group of these interneurons, are the main source of acetylcholine (Ach) in the striatum and provide this brain area with one of the highest contents of this transmitter in the brain (Weiner et al., 1990; Graybiel et al., 1994; Hersch et al., 1994). In spite of their numerical disadvantage, striatal interneurons posses very large axonal and dendritic fields and densely innervate

\footnotetext{
Received May 12, 2000; revised Nov. 20, 2000; accepted Nov. 29, 2000.

This study was supported by Telethon Grant E. 729 (P.C.), by Telethon Grant E. 0930 (A.P.), and by a Ministero dell' Universitá e della Ricerca Scientifica e Tecnologica/Consiglio Nazionale delle Ricerche Grant (legge 95/95; G.B.). We thank Mr. Massimo Tolu for excellent technical assistance.

Correspondence should be addressed to Dr. Paolo Calabresi, Clinica Neurologica, Dipartimento di Neuroscienze, Università di Roma "Tor Vergata," Via di Tor Vergata 135, 00133 Rome, Italy. E-mail: calabre@uniroma2.it.

Copyright (C) 2001 Society for Neuroscience 0270-6474/01/211393-08\$15.00/0
}

on striatal cholinergic cells, as demonstrated by the findings that the membrane depolarization produced by this pharmacological agent was prevented by bath and intracellular application of two inhibitors of soluble guanylyl cyclase and was mimicked and occluded by zaprinast, a cGMP phosphodiesterase inhibitor. Finally, intracellular $\mathrm{Rp}-8-\mathrm{Br}-\mathrm{cGMPS}$, a protein kinase $\mathrm{G}$ (PKG) inhibitor, blocked the hydroxylamine-induced membrane depolarization of cholinergic interneurons, whereas both okadaic acid and calyculin A, two protein phosphatase inhibitors, enhanced it, indicating that intracellular PKG and phosphatases oppositely regulate the sensitivity of striatal cholinergic interneurons to NO. The characterization of the cellular mechanisms involved in the regulation of striatal interneuron activity is a key step for the understanding of the role of these cells in striatal microcircuitry.

Key words: acetylcholine; basal ganglia; brain slices; intracellular recordings; NOS-positive interneurons; protein phosphatases

virtually all neuronal species within this nucleus (Kawaguchi, 1992, 1993; Kawaguchi et al., 1995).

Experimental evidence obtained during microdialysis studies in vivo suggested that NO might be involved in the control of Ach release in the striatum. Few and conflicting data, however, have been collected so far on this interesting issue. In particular, some authors reported that NO donors cause a pronounced increase in striatal Ach release (Prast and Philippu, 1992; Guevara-Guzman et al., 1994; Prast et al., 1995, 1998), which has been attributed, at least in part, to a concomitant increased release of glutamate. Various NO donors, in fact, have been found to be ineffective in triggering Ach release from the striatum in the presence of antagonists of glutamate receptors (Prast et al., 1998). Other studies, however, reported that the release of striatal Ach was unaffected by NO (Sandor et al., 1995) and also that endogenous NO attenuated rather than favored NMDA-induced Ach release (Ikarashi et al., 1998).

Indeed, these results do not allow conclusions to be drawn on the possible interaction between NO and cholinergic cells within the striatum. In the attempt to clarify this issue, in the present study we used an electrophysiological approach in vitro to study the effects of NO on striatal cholinergic interneurons recorded intracellularly.

\section{MATERIALS AND METHODS}

Preparation and maintenance of the corticostriatal slices. Male Wistar rats (2-3 months) were used for the electrophysiological experiments. Prep- 
aration and maintenance of the slices have been described in detail previously (Calabresi et al., 1992, 1999a). In brief, animals were killed under ether anesthesia by cervical dislocation, the brain was quickly removed, and corticostriatal coronal slices (200- to $300-\mu$ m-thick) were cut, from tissue blocks, with the use of a vibratome. Slices were maintained at $31^{\circ} \mathrm{C}$ in an oxygenated solution for $\sim 30 \mathrm{~min}$. A single slice was then transferred into a recording chamber and fully submerged in a continuously flowing Krebs' solution $\left(30^{\circ} \mathrm{C}, 3 \mathrm{ml} / \mathrm{min}\right)$ gassed with $95 \%$ $\mathrm{O}_{2}$ and $5 \% \mathrm{CO}_{2}$. The composition of the solution was (in $\mathrm{mM}$ ): $126 \mathrm{NaCl}$, $2.5 \mathrm{KCl}, 1.3 \mathrm{MgCl}_{2}, 1.2 \mathrm{NaH}_{2} \mathrm{PO}_{4}, 2.4 \mathrm{CaCl}_{2}, 10$ glucose, and 18 $\mathrm{NaHCO}_{3}$. In some experiments choline chloride was used to replace $\mathrm{Na}^{+}$chloride. In these experiments $\mathrm{Na}^{+}$chloride was reduced to $30 \%$ (38 mM).

Electrophysiological recordings. In all the electrophysiological experiments the intracellular recording electrodes were filled with $2 \mathrm{M} \mathrm{KCl}$ (30-60 M $\Omega$ ). An Axoclamp 2A (Axon Instruments, Foster City, CA) amplifier was used for both current- and voltage-clamp recordings. In single-electrode voltage-clamp mode the switching frequency was $3 \mathrm{kHz}$. The headstage signal was continuously monitored on a separate oscilloscope. Traces were displayed on an oscilloscope and stored in a digital system.

Morphological and histochemical characterization of cholinergic interneurons. In some experiments, for simultaneous optical and electrical recordings, the tip of the recording electrode was filled with a solution of 2 mu fura-2 (pentapotassium salt; Molecular Probes, Leiden, The Netherlands) and $100 \mathrm{~mm} \mathrm{KCl,} \mathrm{whereas} \mathrm{the} \mathrm{shank} \mathrm{was} \mathrm{filled} \mathrm{with} \mathrm{a} 2 \mathrm{M} \mathrm{KCl}$ solution. After cell impalement, cells $(n=64)$ were loaded with fura- 2 by injecting, through the recording electrode, $0.1-0.5 \mathrm{nA}$ negative current for 10-15 min. In these cases, the recording chamber was mounted on the stage of an upright microscope (Axioscop FS, Zeiss), equipped with a $60 \times$ water immersion objective (Zeiss). Excitation light passed through a shutter and was filtered at 340 and $380 \mathrm{~nm}$. Emission light was filtered by a long-pass barrier filter $(470 \mathrm{~nm})$ and detected by a CCD camera (Photonic Science, East Sussex, UK). Images were stored and analyzed with a software (IonVision; ImproVision, Birmingham, UK) running on PowerMac 8100. Ratio images were calculated from pairs of 340 and 380 $\mathrm{nm}$ images after background fluorescence was subtracted (backgrounds were acquired from regions free of dye fluorescence). Ratiometric measurements were converted into intracellular calcium concentration values (Grynkiewicz et al., 1985; Pisani et al., 1999).

In other experiments $(n=46)$ biocytin was used in the intracellular electrode to stain the neurons. In these cases, biocytin at concentration of $2-4 \%$ was added to a $2 \mathrm{M} \mathrm{KCl}$ pipette solution. Slices containing neurons stained with biocytin were fixed in $4 \%$ paraformaldehyde in 0.1 $\mathrm{M}$ phosphate buffer (PB) overnight at $4^{\circ} \mathrm{C}$. After incubation in $\mathrm{PB}$ containing sucrose $30 \%$ in $0.1 \mathrm{M} \mathrm{PB}$ for $3 \mathrm{hr}$, the slices were frozen and further resectioned in a cryostat at $40 \mu \mathrm{m}$ thickness. Free-floating sections were incubated with fluorescein isothiocyanate (FITC) conjugated to avidin (Sigma, St. Louis, MO; diluted 1:200 in PBS containing 0.1\% Triton X-100) overnight at $4^{\circ} \mathrm{C}$. The sections were then washed in PB several times and mounted on slides with glycerol in PB (1:3). The sections were observed and photographed in a fluorescence microscope (Leitz, Wetzlar, Germany) using epifluorescence B-2E (barrier filter, 520-560) for FITC to examine biocytin-positive cells. Selected sections, in which a large aspiny neuron had been identified, were further processed for double staining of biocytin and choline acetyltransferase (ChAT) immunoreactivity. The sections were removed from the slides, and after washing in $\mathrm{PB}$, incubated with a rat monoclonal antibody against ChAT (Boehringer Mannheim, Mannheim, Germany; 1:250) in $\mathrm{PB}$ containing $10 \%$ normal goat serum and $2 \%$ bovine serum albumin, for $3 \mathrm{hr}$ at room temperature. After washing in $\mathrm{PB}$, the sections were incubated in a mixture containing goat anti-rabbit IgG (Sigma; 1:50) conjugated to tetramethylrhodamine isothiocyanate (TRITC) and avidin-conjugated FITC (1:200) for $2 \mathrm{hr}$ at room temperature. After washing, the sections were mounted on slides with glycerol in PB (1:3). In this case, the slices were observed and photographed in the fluorescence microscope using epifluorescence G-2A (barrier filter, $>590 \mathrm{~nm}$ ) for TRITC, and epifluorescence B-2E (barrier filter, 520-560 nm) for FITC, so that ChAT-immunoreactive neurons were seen in red, and biocytin positive cells in yellow-green. In several cases, sections were further processed to make permanent staining of biocytin-loaded cells.

Data analysis and drug application. Values given in the text and in the figures are mean \pm SEM of changes in the respective cell populations. Wilcoxon's test or Student's $t$ test (for paired and unpaired observations) were used to compare the means and ANOVA was used when multiple comparisons were made against a single control group. Drugs were applied by dissolving them to the final concentration in the saline and by switching the perfusion from control saline to drug-containing saline. Drug solutions entered the recording chamber within $40 \mathrm{sec}$ after a three-way tap had been turned on. In some experiments, however, $1 \mathrm{H}-[1,2,4]$ oxadiazolo[4,3-a]quinoxalin-1-one (ODQ), 4-H-Bromo-1,2,4oxadiazolo(3,4-d)benz(b)oxazin-1-one (NS 2028), or guanosine $3^{\prime}, 5^{\prime}$ cyclic monophosphorothioate, 8-bromo, Rp-isomer (Rp-8-Br-cGMPS) were applied intracellularly through the recording electrode, as previously reported (Calabresi et al., 1999a). Hydroxylamine was from Merck (Darmstadt, Germany). 6-cyano-7-nitroquinoxaline-2,3-dione (CNQX), (+)-MK 801 maleate (MK-801), ODQ, SCH 23390, $(R S)$ - $\alpha$-methyl-4carboxyphenylglycine (MCPG), and $S$-nitroso- $N$-acetylpenicillamine (SNAP) were from Tocris Cookson (Bristol, UK). Rp-8-Br-cGMPS was from Calbiochem (La Jolla, CA). Zaprinast (M\&B 22948, 2-Opropoxyphenyl-8-azapurin-6-one) was from Rhône-Poulenc Rorer (Dagenham, UK). Okadaic acid, 4-H-Bromo-1,2,4-oxadiazolo(3,4d)benz(b)oxazin-1-one (NS 2028), and Calyculin A were from Alexis (Läufelfingen, Switzerland). Bepridil, biocytin, [D--Arg ${ }^{1}, \mathrm{D}-\mathrm{Pro}^{2}$, D-$\operatorname{Trp}^{7,9}$, Leu $^{11}$ ]-SP, nifedipine, 2-Phenyl-4,4,5,5-tetramethylimidazoline-1oxyl 3-oxide (PTIO), and TTX were from Sigma (Rome, Italy). 3', 4'dichlorobenzamil (DCB) was from E. J. Cragoe, Jr (Nacogdoches, TX). $\omega$-agatoxin TK and $\omega$-conotoxin GVIA were from Alomone Labs (Jerusalem, Israel).

\section{RESULTS}

Electrophysiological, morphological, and histochemical properties of striatal cholinergic interneurons

All striatal neurons included in this study were identified as cholinergic interneurons for both their electrophysiological and morphological characteristics. They comprised 46 of 260 cells when electrodes were placed into the striatum without visual control, the remaining neurons having electrophysiological characteristics of spiny neurons. With visual placement of the recording electrode, a further 64 cholinergic interneurons were obtained. The high proportion of cholinergic cells recorded is also attributable to the increased probability to obtain stable recordings (lasting at least $30 \mathrm{~min}$ ) from these large cells compared with the smaller spiny neurons. Membrane properties of these cells closely resembled the membrane properties reported previously for rat cholinergic interneurons (Kawaguchi, 1992; Bennett and Wilson, 1998; Calabresi et al., 1998). The distinguishing features of these neurons were: low resting membrane potential (RMP) $(-58 \pm 4 \mathrm{mV})$ and high input resistance $(180 \pm 60 \mathrm{M} \Omega)$ compared with other striatal neurons, accommodation of action potential discharge and marked afterhyperpolarization, prominent cesium-sensitive decline in hyperpolarizing electrotonic potential $\left(I_{\mathrm{h}}\right.$ cation current), and large somata $(30-60 \mu \mathrm{m})$ with three to five primary dendrites bearing no spines. Thirty cells were further processed for double staining of biocytin and ChAT immunoreactivity. All these neurons, which showed the electrophysiological and morphological characteristics typical of cholinergic interneurons, were found to be positive for ChAT immunoreactivity.

\section{Effects of the NO donors hydroxylamine and SNAP on striatal cholinergic interneurons}

In current-clamp experiments, bath application of the NO donor hydroxylamine (100 $\mu \mathrm{M}, 2-7 \mathrm{~min})$ invariably induced a small but significant membrane depolarization of the recorded striatal cholinergic interneurons. This membrane depolarization averaged $5 \pm 1.4 \mathrm{mV}$ in amplitude and triggered action potentials when the RMP of the cell was sufficiently depolarized. Higher doses of hydroxylamine $(300 \mu \mathrm{M})$ failed to produce more pronounced effects, whereas lower concentrations $(3,10$, and $30 \mu \mathrm{M})$ revealed a dose-dependent action of this agent on membrane potential of 

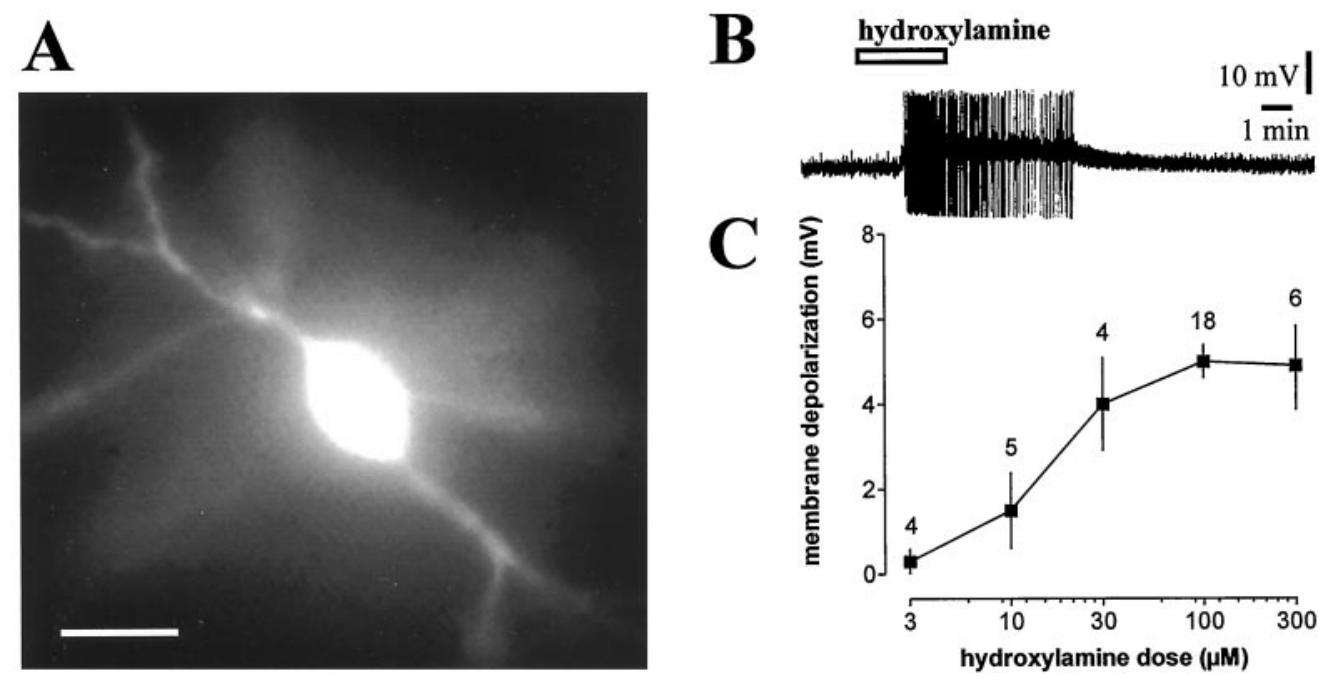

Figure 1. Hydroxylamine depolarizes striatal cholinergic interneurons in a dose-dependent manner. $A$, Morphological identification of a fura-2-filled striatal cholinergic interneuron. Scale bar, $50 \mu \mathrm{m}$. B. Hydroxylamine $(100 \mu \mathrm{M})$ depolarized a striatal cholinergic interneuron and caused action potential firing. Resting level is $-55 \mathrm{mV}$; full action potential height not captured by pen recorder. $C$, The graph shows the dose-response curve for the hydroxylamine-induced membrane depolarization of striatal cholinergic interneurons. In this graph and in the following ones, the number of observations is indicated for each experimental condition.
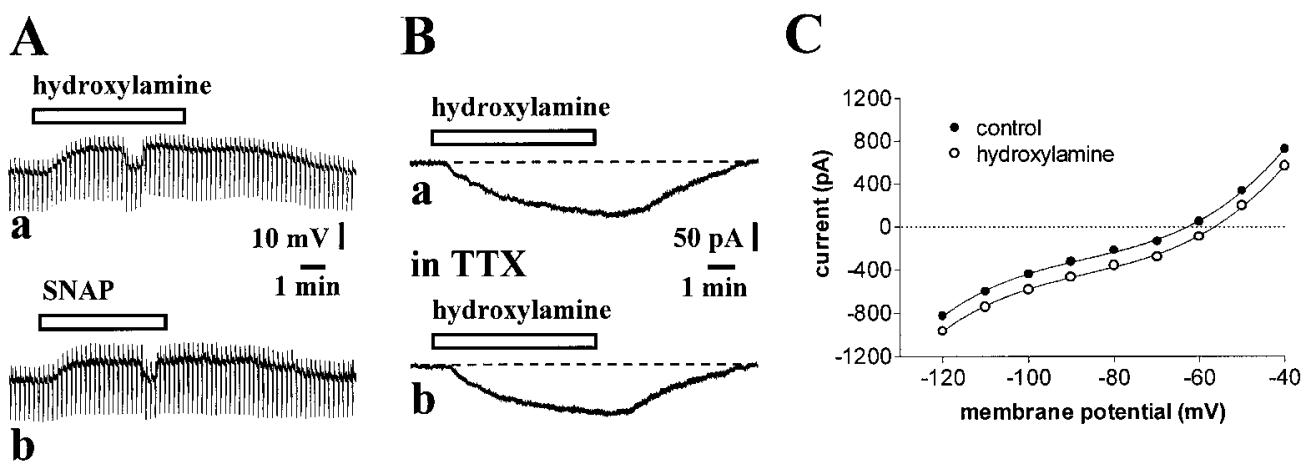

Figure 2. Electrophysiological characteristics of hydroxylamine- and SNAP-induced excitation of striatal cholinergic interneurons. $A$, In a current-clamp experiment, hydroxylamine $(100 \mu \mathrm{M})(a)$ and SNAP $(100 \mu \mathrm{M})(b)$ depolarized a striatal cholinergic interneuron without affecting the apparent input resistance of the cell. To compare the effects of both compounds on this electrophysiological parameter, membrane potential value was returned to the control level by continuous injection of $100 \mathrm{pA}$ negative current. Resting level was $-61 \mathrm{mV}$. Downward deflections are hyperpolarizing electrotonic potentials evoked by rectangular current pulses $(200 \mathrm{pA}, 2 \mathrm{sec})$; their decline after the initial peak reflects the prominent $I_{\mathrm{h}}$ in these cells. $B$, In another striatal interneuron recorded in the voltage-clamp mode, $100 \mu \mathrm{M}$ hydroxylamine produced an inward current $(a)$. This effect persisted unchanged in the presence of $1 \mu \mathrm{M}$ TTX, to block voltage-dependent sodium channels $(b)$. Holding potential was $-60 \mathrm{mV}$. $C$, Current-voltage relationship of a cholinergic interneuron before (open circles) and during $100 \mu \mathrm{M}$ hydroxylamine ( filled circles). The values were calculated by measuring the steady-state current generated by $3 \mathrm{sec}$ voltage steps of progressively increasing and decreasing amplitude. Holding potential was $-60 \mathrm{mV}$.

the recorded striatal cholinergic interneurons (Fig. 1). The effect of $100 \mu \mathrm{M}$ hydroxylamine was mimicked by another NO donor, SNAP (100 $\mu \mathrm{M} ; 3-7 \mathrm{~min} ; p<0.05)(n=5)$, and was fully reversible after 8-15 $\mathrm{min}$ washout of both drugs. Neither hydroxylamine- nor SNAP-induced membrane depolarization of cholinergic cells was coupled to significant changes in intracellular calcium concentration ( $n=52 ; p>0.05$; data not shown). In some experiments, hyperpolarizing current pulses (100-200 pA; 2-3 sec duration; $10 \mathrm{sec}$ interval) were applied to monitor input resistance of the cells. To measure the effects of NO on this electrophysiological parameter, during the hydroxylamine- or SNAP-induced membrane depolarization, membrane potential value was returned to the control level through the injection of continuous negative current. In the recorded cells, the input resistance was not significantly affected by these NO donors $(n=$ 6 for each experimental condition; $p>0.05$; Fig. $2 A$ ).
When the cholinergic interneurons were voltage-clamped at or near the RMP $(-60 \mathrm{mV})$, bath application of hydroxylamine produced an inward shift of the holding current in all the tested neurons $(-140 \pm 25 \mathrm{pA} ; p<0.01 ; n=12)$. This effect persisted unchanged after the application of $1 \mu \mathrm{M}$ TTX, a voltagedependent sodium channel blocker, and recovered within $15 \mathrm{~min}$ of washout (Fig. 2B). TTX was also found to be ineffective on hydroxylamine- $(p>0.05 ; n=6$; Fig. $3 C)$ and SNAP-mediated electrophysiological effects in current-clamp experiments $(p>$ $0.05 ; n=4$; data not shown). In an attempt to uncover the ionic mechanisms for hydroxylamine-induced membrane depolarization/inward current, voltage steps $(3 \mathrm{sec})$ of progressively increasing amplitude were applied in the presence of $1 \mu \mathrm{M}$ TTX to voltage-clamped interneurons before and during the application of this NO donor. The resulting current-voltage relationships were calculated by measuring the steady-state current during each 


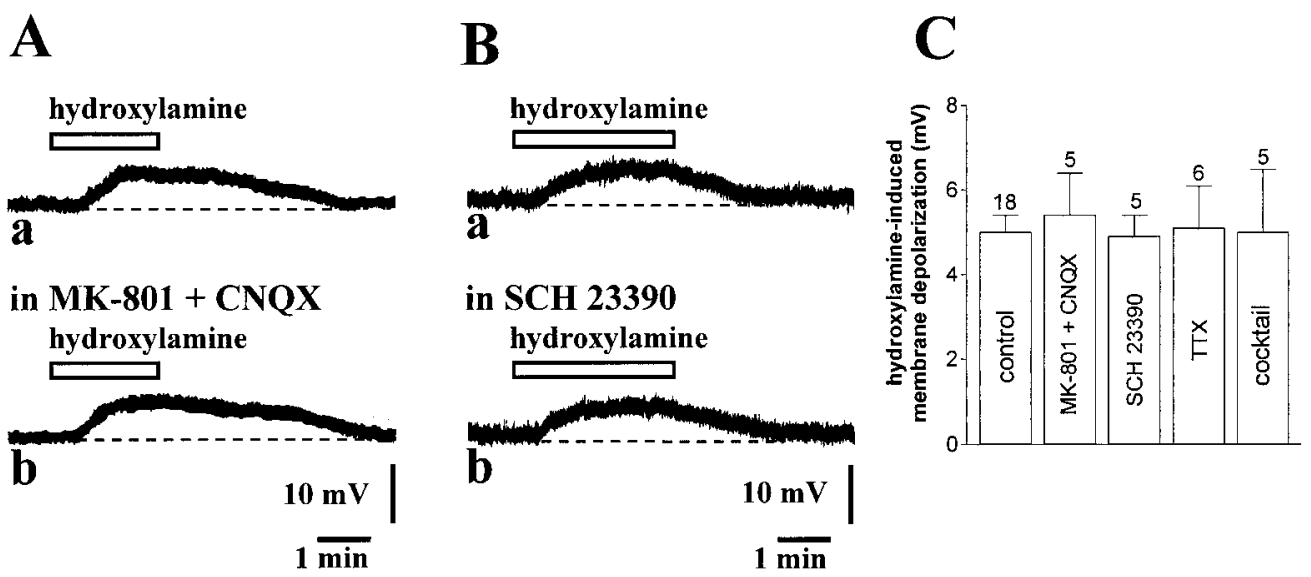

Figure 3. Various receptor antagonists and calcium channel blockers fail to prevent hydroxylamine-induced membrane depolarization of striatal cholinergic interneurons. $A$, The membrane depolarization produced by $100 \mu \mathrm{M}$ hydroxylamine $(a)$ was not altered in the presence of $30 \mu \mathrm{M}$ MK- 801 and $10 \mu \mathrm{M}$ CNQX $(7 \mathrm{~min})$ to block both NMDA and non-NMDA glutamate receptors $(b)$. Resting membrane potential was $-58 \mathrm{mV}$. $B$, Application of the DA D1 receptor antagonist SCH $23390(10 \mu \mathrm{M}, 7 \mathrm{~min})(a)$ failed to affect the membrane response produced in control medium by $100 \mu \mathrm{M}$ hydroxylamine $(b)$. Resting membrane potential was $-58 \mathrm{mV}$. C, Summary of pharmacological experiments on $100 \mu \mathrm{M}$ hydroxylamine-induced membrane depolarization of striatal cholinergic interneurons. Cocktail solution contained: $\omega$-conotoxin GVIA, nifedipine, $\omega$-agatoxin TK, MCPG, and [D--Arg ${ }^{1}$,D--Pro ${ }^{2}$,D--Trp ${ }^{7,9}$, Leu $^{11}$ ]-SP. Concentrations were: MK-801 $30 \mu \mathrm{M}$, CNQX $10 \mu \mathrm{M}$, SCH $2339010 \mu \mathrm{M}$, TTX $1 \mu \mathrm{M}, \omega$-conotoxin GVIA $1 \mu \mathrm{M}$,

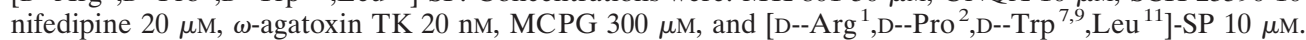

voltage step. As shown in Figure $2 C$, a net inward current was present at all membrane potentials with no apparent reversal potential within the tested voltage range. Although poor space clamp is assumable given the size of these neurons and the limitation of the recording technique, this evidence might indicate that this current does not result, at least in the totality, from the blockade of potassium channels, and might suggests that either an increased sodium and/or calcium conductance with a reversal potential positive to the explored membrane potentials, or the modulation of a membrane ion transporter might be involved.

\section{NO-mediated membrane depolarization of striatal cholinergic interneurons does not depend on the release of endogenous neurotransmitters}

The effect of hydroxylamine on striatal cholinergic interneurons was not inhibited preincubating the slices (5-10 min) with MK$801(30 \mu \mathrm{M})$ plus CNQX $(10 \mu \mathrm{M})$, antagonists of NMDA and non-NMDA glutamate receptors, respectively, suggesting that this effect was not mediated by an increased release of glutamate $(p>0.05 ; n=5$; Fig. $3 A, C)$. Because it has been reported that $\mathrm{NO}$ also increases dopamine release in the striatum both in vitro (Zhu and Luo, 1992; Black et al., 1994; Stewart et al., 1996) and in vivo (Spatz et al., 1995; West and Galloway, 1996, 1997) and that D1-like dopamine receptor activation depolarizes striatal cholinergic interneurons with a time course similar to that one produced by hydroxylamine (Aosaki et al., 1998; Pisani et al., 2000), we also tested the possibility that hydroxylamine caused its effects through the release of endogenous dopamine. In five experiments, therefore, we bath-applied the NO donor after pretreatment of the slices with $10 \mu \mathrm{M} \mathrm{SCH} 23390$ (5-10 min), a D1-like dopamine receptor antagonist able to fully block membrane depolarization of striatal cholinergic neurons induced by dopamine (Aosaki et al., 1998). We found that this pharmacological agent failed to prevent the membrane depolarization of cholinergic interneurons induced by hydroxylamine $(p>0.05)$, ruling out the contribution of endogenous dopamine in this effect (Fig. 3B,C).

NO might favor transmitter release in the striatum indepen- dently of the activation of TTX-sensitive sodium channels and might depolarize cholinergic cells indirectly via glutamate acting on metabotropic glutamate receptors (mGluRs) or via the release of other transmitters. We measured, therefore, the effects of 100 $\mu \mathrm{M}$ hydroxylamine after $10 \mathrm{~min}$ preincubation of the slices in a solution containing $1 \mu \mathrm{M} \omega$-conotoxin GVIA, $20 \mu \mathrm{M}$ nifedipine, and $20 \mathrm{~nm} \omega$-agatoxin TK (to block, respectively, N-type, L-type, and P-type high voltage-activated calcium channels) and $300 \mu \mathrm{M}$ MCPG plus $10 \mu \mathrm{M}\left[\mathrm{D}--\mathrm{Arg}^{1}\right.$,D--Pro ${ }^{2}$,D--Trp ${ }^{7,9}$, Leu $^{11}$ ]-SP (to block mGluRs and substance $\mathrm{P}$ receptors, respectively). Noticeably, $\omega$-conotoxin GVIA has already been found to block synaptic inputs to striatal cholinergic interneurons (Pisani et al., 2000), and MCPG and [D--Arg $\left.{ }^{1}, \mathrm{D}--\mathrm{Pro}^{2}{ }^{2} \mathrm{D}-\mathrm{D}^{-} \mathrm{Trp}^{7,9}, \mathrm{Leu}^{11}\right]-\mathrm{SP}$ prevented in previous studies the depolarizing effects mediated by mGluR and substance $\mathrm{P}$ receptor activation on these cells (Aosaki and Kawaguchi, 1996; Takeshita et al., 1996). This pharmacological treatment, however, failed to affect the amplitude of the NOinduced membrane depolarization of striatal interneurons $(p>$ $0.05 ; n=5$; Fig. $3 C$ ). These data, coupled to the observation that TTX $(1 \mu \mathrm{M})$ did not affect the membrane responses to $100 \mu \mathrm{M}$ hydroxylamine (see above) strengthen the conclusion that NO depolarizes striatal cholinergic interneurons through a direct postsynaptic action.

\section{Effects of low sodium-containing solution and of sodium transporter blockers on hydroxylamine- induced membrane depolarization of striatal cholinergic interneurons}

To investigate whether the membrane depolarization produced by NO donors was mediated by sodium influx, in some experiments we reduced this extracellular ion by substituting it with choline chloride. As shown in Figure 4, the $100 \mu \mathrm{M}$ hydroxylamine-evoked membrane depolarization recorded in a solution containing $126 \mathrm{~mm} \mathrm{NaCl}$ showed a remarkable reduction when the perfusing solution was switched to a solution containing $38 \mathrm{~mm} \mathrm{NaCl}(n=6)$, indicating that sodium ions are the major carrier of this depolarization.

Sodium transport and some sodium channels are sensitive to the amiloride derivative DCB (Wacholtz et al., 1993; Calabresi et 

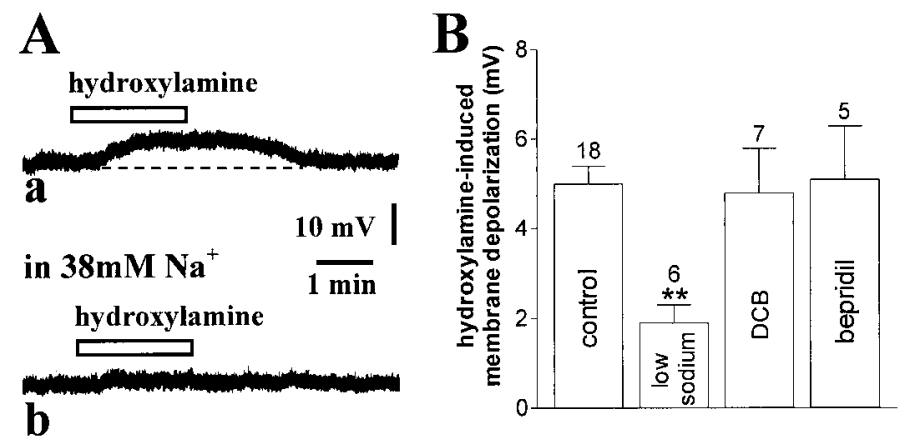

Figure 4. Hydroxylamine-induced membrane depolarization is significantly attenuated by low sodium-containing external solution but not by sodium exchanger blockers. $A$, In this current-clamp experiment, the membrane depolarization produced by $100 \mu \mathrm{M}$ hydroxylamine $(a)$ was blocked by 7 min perfusion of the slices with a solution containing $38 \mathrm{~mm}$ sodium ions $(b)$. Resting membrane potential was $-61 \mathrm{mV}$. B, Summary of experiments on hydroxylamine $(100 \mu \mathrm{M})$-induced membrane depolarization of striatal cholinergic interneurons. Concentration of both DCB and bepridil was $100 \mu \mathrm{M}\left({ }^{*} p<0.01\right)$.

al., 1999b) and to bepridil (Stys et al., 1992; Kiedrowski et al., 1994). We tested, therefore, these two pharmacological agents on the membrane responses induced by bath application of $100 \mu \mathrm{M}$ hydroxylamine on striatal cholinergic interneurons. Preincubation of the slices with either DCB (5-10 min; $100 \mu \mathrm{M} ; n=7)$ or bepridil (5-10 $\min ; 100 \mu \mathrm{M} ; n=5$ ) produced per se no change in the RMP, and apparent input resistance of the recorded neurons and failed to affect the membrane responses evoked by the application of hydroxylamine $(100 \mu \mathrm{M} ; 2-7 \mathrm{~min})(p>0.05$ for both experimental conditions) (Fig. $4 B$ ). Noticeably, at the concentrations used in this study, both DCB and bepridil neither affected RMP of striatal spiny neurons recorded in vitro. During in vitro ischemia, however, the ability of both agents in inhibiting sodium-calcium exchanger was unmasked (Calabresi et al., 1999b). This observation confirms that although DCB- and bepridilsensitive transporters do not seem involved in the excitatory action of NO in striatal cholinergic cells, $100 \mu \mathrm{M}$ DCB and $100 \mu \mathrm{M}$ bepridil are able to target ion transporters in our slice preparation.

\section{Requirement of postsynaptic cGMP elevation in the hydroxylamine-induced membrane depolarization of striatal cholinergic interneurons}

NO elevates intracellular cGMP levels by stimulating soluble guanylyl cyclase (sGC) activity. Thus, to address the involvement of cGMP in the electrophysiological effects produced by hydroxylamine, we first investigated whether the pharmacological inhibition of sGC was able to prevent the membrane depolarization caused by this NO donor. Preincubation of the slices with $10 \mu \mathrm{M}$ ODQ (5-10 min), a selective inhibitor of sGC, did not cause any change in the membrane potential of the recorded striatal interneurons $(n=12)$ but fully blocked the membrane depolarization produced in these cells by $100 \mu \mathrm{M}$ hydroxylamine $(p<0.01)$. This pharmacological inhibition was reversible after a period of washout of 10-15 min. Interestingly, ODQ $(100 \mu \mathrm{M})$ strongly reduced hydroxylamine-induced membrane depolarization also when this sGC inhibitor was applied intracellularly through the recording pipette $(15 \mathrm{~min})$, demonstrating that NO produces its effects by stimulating sGC activity in cholinergic interneurons themselves $(n=7 ; p<0.01$; Fig. $5 C)$. Similar results were obtained by applying intracellularly (15 min) NS 2028 (50 $\mu \mathrm{M})$, another spe- cific inhibitor of sGC $(n=4 ; p<0.01$; Fig. $5 C)$. Although neither ODQ nor NS 2028 are membrane-impermeable compounds, these data, together with those obtained with TTX, calcium channel blockers and receptor antagonists, are all supportive of the idea that the enzymes mediating the response to NO are located in cholinergic interneurons themselves.

To further confirm that an increase in cGMP levels mediated the physiological effects of hydroxylamine, we tested whether the inhibition of cGMP breakdown by zaprinast could mimic and occlude the physiological responses of this NO donor. Zaprinast is a rather selective inhibitor of cGMP phosphodiesterase, which has been already demonstrated to cause both significant elevation of cGMP levels in extracts of striatal slices and long-term depression of the efficacy of excitatory synaptic transmission in striatal projection cells (Calabresi et al., 1999a). In current-clamp recordings, bath application of this pharmacological agent (30 $\mu \mathrm{M}, 3-5$ min) caused a membrane depolarization in five of eight striatal cholinergic interneurons. This membrane depolarization had similar amplitude than that one recorded in the presence of 100 $\mu \mathrm{M}$ hydroxylamine $(4.8 \pm 1 \mathrm{mV} ; p<0.05$; Fig. $5 B a)$ and was coupled with no change in the apparent input resistance of the recorded neurons (data not shown). Moreover, in a different manner than the effects on excitatory transmission in striatal projection neurons, the electrophysiological effects of zaprinast on cholinergic cells were quickly reversible at the wash-out of the drug. The ability of zaprinast in producing per se the reported electrophysiological effects might suggest the existence of a resting NO tone in striatal slices. To address this issue, therefore, we studied the effects of the NO scavenger PTIO (30 $\mu \mathrm{M}$; 5-7 min; $n=4$ ) on the resting membrane potential of striatal cholinergic cells. This agent produced no effect on these cells, suggesting that endogenous NO is unable to depolarize cholinergic cells in the absence of a concomitant inhibition of cGMP breakdown (data not shown). In six experiments, $30 \mu \mathrm{M}$ zaprinast was added to the bathing solution during the steady state of the membrane depolarization produced by $100 \mu \mathrm{M}$ hydroxylamine, and in other four experiments the application of zaprinast preceded that one of hydroxylamine. When hydroxylamine was applied first, the subsequent application of zaprinast did not enhance the membrane depolarization caused by hydroxylamine $(6 \pm 0.8$ vs $6.5 \pm 0.5 \mathrm{mV}$; $p>0.05)$. Similarly, when zaprinast preceded the application of hydroxylamine, this latter compound produced only a small potentiation of the depolarizing effect of zaprinast alone $(5 \pm 0.8$ vs $5.8 \pm 1 \mathrm{mV} ; p>0.05$ ) (Fig. 5B). These data show that hydroxylamine- and zaprinast-induced membrane depolarizations of striatal cholinergic cells were mutually occlusive, that is, during the hydroxylamine- or zaprinast-induced membrane depolarization, further depolarizations were not inducible by, respectively, zaprinast or hydroxylamine.

\section{Role of protein kinase $\mathrm{G}$ and protein phosphatases in the hydroxylamine-induced membrane depolarization of striatal cholinergic interneurons}

Intracellular elevation of cGMP levels results in the stimulation of the cGMP-dependent protein kinase (PKG). This effector, in turn, modulates the function of a series of cellular substrates by increasing their phosphorylation state. Therefore, to test whether PKG stimulation was involved in the action of NO on cholinergic cells of the striatum, we intracellularly applied $1 \mu \mathrm{M} \mathrm{Rp}-8-\mathrm{Br}-$ cGMPS, a selective inhibitor of this kinase. This agent did not affect resting membrane potential and input resistance of the recorded cells, but prevented their membrane depolarization 
Figure 5. Hydroxylamine-induced membrane depolarization of striatal cholinergic interneurons requires cGMP elevation. $A$, The pharmacological blockade of sCG by ODQ $(5 \mathrm{~min}, 10$ $\mu \mathrm{M})$ (b) fully prevented the membrane depolarization of a cholinergic interneuron produced by $100 \mu \mathrm{M}$ hydroxylamine $(a)$. This inhibition was reversible after $15 \mathrm{~min}$ wash of this compound $(c)$. Resting membrane potential was $-60 \mathrm{mV}$. B. The cGMP phosphodiesterase inhibitor zaprinast $(30 \mu \mathrm{M})$ produced a membrane depolarization of another neuron and prevented further depolarization when $100 \mu \mathrm{M}$ hydroxylamine was added $(a)$. After $10 \mathrm{~min}$ wash of both pharmacological agents, the ability of $100 \mu \mathrm{M}$ hydroxylamine to depolarize the
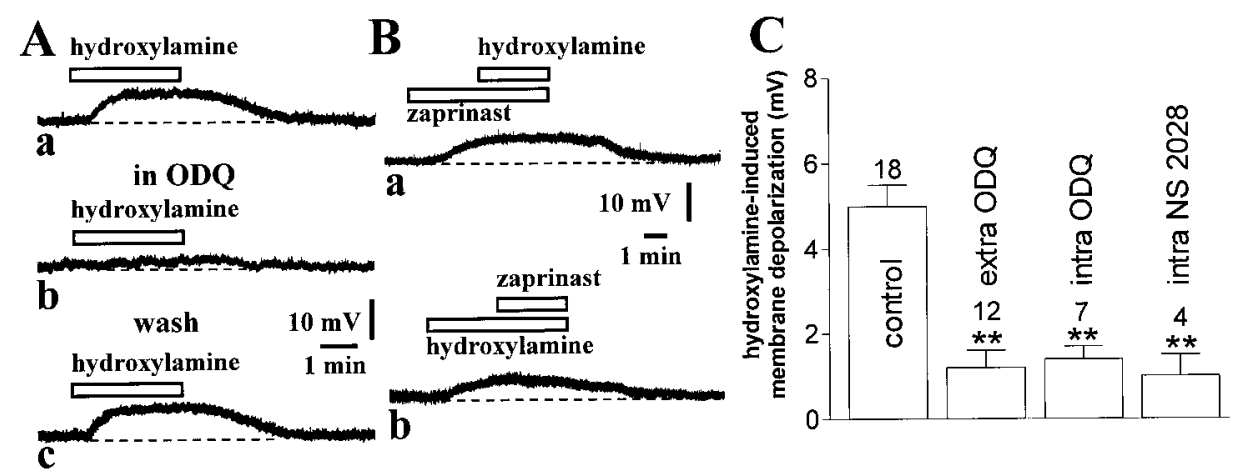
recorded cell was restored and $30 \mu \mathrm{M}$ zaprinast failed to produce significant depolarization when applied in the presence of this NO donor $(b)$. Resting membrane potential was $-62 \mathrm{mV}$. C, Summary of experiments on hydroxylamine $(100 \mu \mathrm{M})$-induced membrane depolarization of striatal cholinergic interneurons. Concentrations were (in $\mu \mathrm{M}$ ): extracellular ODQ 10, intraelectrode ODQ 100, and intraelectrode NS $202850(* * p<0.01)$.

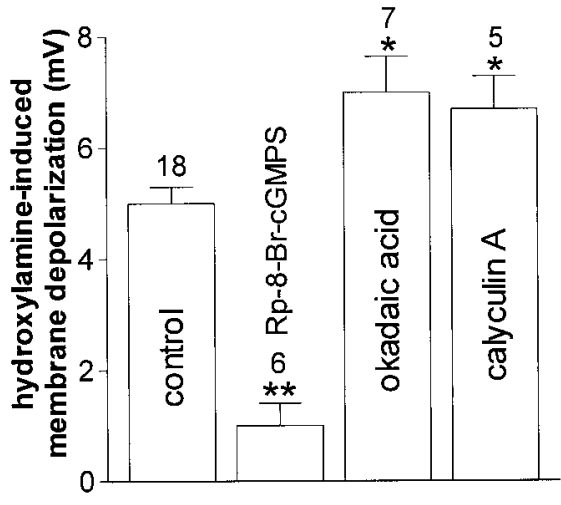

Figure 6. Role of intracellular PKG and protein phosphatases in the hydroxylamine-induced membrane depolarization of striatal cholinergic cells. The histogram shows the effects of the pharmacological blockade of postsynaptic PKG and protein phosphatases on the membrane depolarization produced by $100 \mu \mathrm{M}$ hydroxylamine (see Results for details). Concentrations were: Rp-8-Br-cGMPS $1 \mu \mathrm{M}$, okadaic acid $30(n=3) 100$ $\mathrm{nM}(n=4)$, and calyculin A $100 \mathrm{nM}(* p<0.05 ; * *<0.01)$.

after the application of $100 \mu \mathrm{M}$ hydroxylamine. These observations suggest that an increased phosphorylation of critical PKGsensitive substrates mediates the effects of NO on these striatal cells. Because the phosphorylation state of cellular substrates also depends on the action of specific phosphatases (Greengard et al., 1999), we also studied the effects of either okadaic acid (30-100 $\mathrm{nM})$ or calyculin A (100 nM) on hydroxylamine-induced membrane depolarization. These two pharmacological agents are rather selective inhibitors of protein phosphatase-1 (PP-1) and protein phosphatase-2A (PP-2A), enzymes that counteract the action of various intracellular kinases. In the presence of $1 \mu \mathrm{M}$ TTX, both these compounds, bath-applied 5-7 min before the further application of hydroxylamine, did not produce per se any change in the RMP and input resistance of the recorded cell, but slightly enhanced the amplitude of hydroxylamine-induced membrane depolarization in striatal cholinergic interneurons, suggesting that in physiological conditions PP-1 and/or PP-2A limit the NO-stimulated PKG activity (Fig. 6).

\section{DISCUSSION}

The present study demonstrates that NO enhances membrane excitability of cholinergic interneurons. NO-induced membrane depolarization of striatal cholinergic interneurons is not secondary to the release of excitatory amino acids, dopamine, substance
$\mathrm{P}$, or other putative excitatory transmitters, because it persisted unchanged in the presence of glutamate, dopamine, and substance P receptor antagonists, as well as in the presence of TTX and calcium channel blockers. This action requires intracellular cGMP elevation via sGC activity stimulation. Accordingly, the pharmacological inhibition of sGC fully prevented hydroxylamine-induced membrane depolarization of striatal cholinergic cells, whereas the inhibition of cGMP breakdown mimicked and occluded this electrophysiological effect. In addition, hydroxylamine-induced membrane depolarization was prevented by intracellular injection of either sGC or PKG inhibitors, indicating that NO likely acts as an anterograde transmitter on striatal cholinergic interneurons, leading to cGMP-dependent stimulation of PKG. These electrophysiological data are in good agreement with ultrastructural and immunocytochemical studies, demonstrating that NOS-positive terminals do synapse on cholinergic cells in the striatum (Vuillet et al., 1992) and that intracellular cGMP synthesis occurs in cholinergic cells but not in dopaminergic or glutamatergic presynaptic fibers of the rat striatum in response to NO (De Vente et al., 2000). It should be noted, however, that the compounds used in this study to block sGC or PKG are all membrane-permeable and, therefore, it is also possible that the enzymes mediating the response of cholinergic cells to NO are located in other cellular subtypes of our slice preparation.

The evidence that in striatal cholinergic interneurons protein phosphatase inhibitors significantly increased the membrane responses to hydroxylamine suggests the existence of a functional antagonism between PKG and phosphatases in determining the sensibility to NO in these cells. It is therefore conceivable that PKG and protein phosphatases oppositely regulate the phosphorylation state of common intracellular substrates, important for the physiological action of NO.

The experiments showing that the NO-induced membrane depolarization of striatal cholinergic interneurons is significantly reduced in low sodium-containing solution strongly suggest that sodium ions are the main carrier of this depolarization. We failed, however, to identify the channel subtypes involved in this action. The evidence that blockade of N-type, L-type, and P-type voltage-dependent calcium channels had no effect on the hydroxylamine-mediated electrophysiological actions seems to rule out the involvement of these channels. The estimation of the reversal potential of the hydroxylamine-mediated current is probably hampered by space-clamp errors. Nevertheless, our results showing that the hydroxylamine-mediated current does not re- 
verse polarity close to the potassium equilibrium potential (approximately $-100 \mathrm{mV}$ ) do not support the idea that the modulation of potassium conductances is responsible for this electrophysiological effect. It is however possible that the modulation of potassium conductances might be involved in the electrophysiological action of NO as part of a mixed current. Chloride channels are also potential candidates in the generation of this current. Because of the high concentration of chloride ions in the recording pipette, the chloride equilibrium potential is presumably more positive to the average resting membrane potential of cholinergic interneurons and, therefore, an increased permeability to these ions would contribute to the generation of an inward current. Experiments using patch-clamp recordings from isolated neurons appear more appropriate to clarify all these issues.

This study demonstrates that NO is able to influence the physiological activity of central neurons by causing membrane depolarization. Although it has been found that NO acts as a neurotransmitter in the PNS (Briggs, 1992), in the CNS it revealed so far only "nonconventional" actions. In particular, it has been reported that this agent is involved in the induction of hippocampal long-term potentiation (LTP) (O'Dell et al., 1991; Schuman and Madison, 1991), cerebellar (Crepel and Jaillard, 1990; Böhme et al., 1991; Daniel et al., 1993) and striatal longterm depression (Calabresi et al., 1999a), and increases, presumably through an action on presynaptic nerve terminals, the release of various neurotransmitters in the hippocampus (Nei et al., 1996) and basal ganglia (Kuriyama and Ohkum, 1995; Bogdanov and Wurtman, 1997). The ability to increase neurotransmitter release supports the idea that this agent mainly acts as retrograde transmitter and, although this issue remains controversial, it has been reported that injection of inhibitors of NOS into the postsynaptic cell prevented LTP formation (O'Dell et al., 1991; Schuman and Madison, 1991), as well as presynaptic injection of NO-absorbing agents (Arancio et al., 1996).

Several physiological activities have been postulated for NO in the striatum. NO, in fact, is thought to be released by NOSpositive interneurons to control local blood flow in response to cortical or pallidal inputs (Kawaguchi, 1993; Kawaguchi et al., 1995) and also influences striatal neuron activity by interacting directly with glutamate receptors (Manzoni et al., 1992). Moreover, striatal NO increases gap junction permeability in spiny neurons recorded in vitro (O'Donnell and Grace, 1997) and modulates the activity of striatal feedback pathways involved in regulating dopamine neurons in the substantia nigra (West and Grace, 2000). Together with the report of the present work, these studies indicate that NO exerts a complex control of striatal function in processes which may or may not be involved in modulating excitatory synaptic transmission.

\section{REFERENCES}

Aosaki T, Kawaguchi Y (1996) Actions of substance P on rat neostriatal neurons in vitro. J Neurosci 16:5141-5153.

Aosaki T, Kiuchi K, Kawaguchi Y (1998) Dopamine D1-like receptor activation excites rat striatal large aspiny neurons in vitro. J Neurosci 18:5180-5190.

Arancio O, Kiebler M, Lee CJ, Lev-Ram V, Tsien RY, Kandel ER, Hawkins RD (1996) Nitric oxide acts directly in the presynaptic neuron to produce long-term potentiation in cultured hippocampal neurons. Cell 87:1025-1035.

Bennett BD, Wilson CJ (1998) Synaptic regulation of action potential timing in neostriatal cholinergic interneurons. J Neurosci 18:8539-8549.

Black MD, Matthews EK, Humphrey PP (1994) The effects of a photosensitive nitric oxide donor on basal and electrically-stimulated dopamine efflux from the rat striatum in vitro. Neuropharmacology 33:1357-1365.
Bogdanov MB, Wurtman RJ (1997) Possible involvement of nitric oxide in NMDA-induced glutamate release in the rat striatum: an in vivo microdialysis study. Neurosci Lett 221:197-201.

Böhme GA, Bon C, Stutzmann JM, Doble A, Blanchard JC (1991) Possible involvement of nitric oxide in long-term potentiation. Eur J Pharmacol 199:379-381.

Briggs CA (1992) Potentiation of nicotinic transmission in the rat superior cervical sympathetic ganglion: effects of cyclic GMP and nitric oxide generators. Brain Res 573:139-146.

Calabresi P, Maj R, Pisani A, Mercuri NB, Bernardi G (1992) Longterm synaptic depression in the striatum: physiological and pharmacological characterization. J Neurosci 12:4224-4233.

Calabresi P, Centonze D, Pisani A, Sancesario G, North RA, Bernardi G (1998) Muscarinic IPSPs in rat striatal cholinergic interneurones. J Physiol 510:421-427.

Calabresi P, Gubellini P, Centonze D, Sancesario G, Morello M, Giorgi M, Pisani A, Bernardi G (1999a) A critical role of the nitric oxide/ cGMP pathway in corticostriatal long-term depression. J Neurosci 19:2489-2499.

Calabresi P, Marfia GA, Amoroso S, Pisani A, Bernardi G (1999b) Pharmacological inhibition of the $\mathrm{Na}^{+} / \mathrm{Ca}^{2+}$ exchanger enhances depolarizations induced by oxygen/glucose deprivation but not responses to excitatory amino acids in rat striatal neurons. Stroke 30:1687-1693.

Crepel F, Jaillard D (1990) Protein kinases, nitric oxide and long-term depression of synapses in the cerebellum. NeuroReport 1:133-136.

Daniel H, Hemart N, Jaillard D, Crepel F (1993) Long-term depression requires nitric oxide and guanosine $3^{\prime}: 5^{\prime}$ cyclic monophosphate production in rat cerebellar Purkinje cells. Eur J Neurosci 5:1079-1082.

De Vente J, Markerink-van Ittersum M, van Abeelen J, Emson PC, Axer H, Steinbusch HWM (2000) NO-mediated cGMP synthesis in cholinergic neurons in the rat forebrain: effects of lesioning dopaminergic or serotonergic pathways on nNOS and cGMP synthesis. Eur J Neurosci 12:507-519.

Graybiel AM, Aosaki T, Flaherty A, Kimura M (1994) The basal ganglia and adaptive motor control. Science 265:1826-1831.

Greengard P, Allen PB, Nairn AC (1999) Beyond the dopamine receptor: the DARPP-32/protein phosphatase-1 cascade. Neuron 23:435-447.

Grynkiewicz G, Poenie M, Tsien RY (1985) A new generation of $\mathrm{Ca}^{2+}$ indicators with greatly improved fluorescence properties. J Biol Chem $260 \cdot 3440-3450$.

Guevara-Guzman R, Emson PC, Kendrick KM (1994) Modulation of in vivo striatal transmitter release by nitric oxide and cyclic GMP. J Neurochem 62:807-810.

Hersch SM, Gutekunst CA, Rees HD, Heilman CJ, Levey AI (1994) Distribution of $\mathrm{m} 1-\mathrm{m} 4$ muscarinic receptor proteins in the rat striatum: light and electron microscopic immunocytochemistry using subtypespecific antibodies. J Neurosci 14:3351-3363.

Ikarashi Y, Takahashi A, Ishimaru H, Shiobara T, Maruyama Y (1998) The role of nitric oxide in striatal acetylcholine release induced by $N$-methyl-D-aspartate. Neurochem Int 33:255-261.

Kawaguchi Y (1992) Large aspiny cells in the matrix of the rat neostriatum in vitro: physiological identification, relation to the compartments and excitatory postsynaptic currents. J Neurophysiol 67:1669-1682.

Kawaguchi Y (1993) Physiological, morphological, and histochemical characterization of three classes of interneurons in rat neostriatum. J Neurosci 13:4906-4923.

Kawaguchi Y, Wilson CJ, Augood SJ, Emson PC (1995) Striatal interneurons: chemical, physiological and morphological characterization. Trends Neurosci 18:527-535.

Kiedrowski L, Brooker G, Costa E, Wroblewski JT (1994) Glutamate impairs neuronal calcium extrusion while reducing sodium gradient. Neuron 12:295-300.

Kuriyama K, Ohkum S (1995) Role of nitric oxide in central synaptic transmission: effects on neurotransmitter release. Jpn J Pharmacol 69:1-8.

Manzoni O, Prezeau L, Marin P, Deshager S, Bockaert J, Fagni L (1992) Nitric oxide-induced blockade of NMDA receptors. Neuron 8:653-662.

Nei K, Matsuyama S, Shuntoh H, Tanaka C (1996) NMDA receptor activation induces glutamate release through nitric oxide synthesis in guinea pig dentate gyrus. Brain Res 728:105-110.

O'Dell TJ, Hawkins RD, Kandel ER, Arancio O (1991) Tests of the roles of two diffusible substances in long-term potentiation: evidence for nitric oxide as a possible early retrograde messenger. Proc Natl Acad Sci USA 88:11285-11289.

O'Donnell P, Grace AA (1997) Cortical afferents modulate striatal gap junction permeability via nitric oxide. Neuroscience 76:1-5.

Pisani A, Calabresi P, Centonze D, Marfia GA, Bernardi G (1999) Electrophysiological recordings and calcium measurements in striatal large aspiny interneurons in response to combined $\mathrm{O}_{2} /$ glucose deprivation. J Neurophysiol 81:2508-2516.

Pisani A, Bonsi P, Centonze D, Calabresi P, Bernardi G (2000) Activation of D2-like dopamine receptors reduces synaptic inputs to striatal cholinergic interneurons. J Neurosci 20:RC69(1-6). 
Prast H, Philippu A (1992) Nitric oxide releases acetylcholine in the basal forebrain. Eur J Pharmacol 216:139-140.

Prast H, Fischer H, Werner E, Werner-Felmayer G, Philippu A (1995) Nitric oxide modulates the release of acetylcholine in the ventral striatum of the freely moving rat. Eur J Pharmacol 352:67-73.

Prast H, Tran MH, Fischer H, Philippu A (1998) Nitric oxide-induced release of acetylcholine in the nucleus accumbens: role of cyclic GMP, glutamate, and GABA. J Neurochem 71:266-273.

Sandor NT, Brassai A, Puskas A, Lendvai B (1995) Role of nitric oxide in modulating neurotransmitter release from rat striatum. Brain Res Bull 36:483-486.

Schuman EM, Madison DV (1991) A requirement for the intercellular messenger nitric oxide in long-term potentiation. Science 254:1503-1506.

Spatz M, Yasuma Y, Strasser A, Kawai N, Stanimirovic D, McCarron R (1995) Modulation of striatal dopamine release in cerebral ischemia by L-arginine. Neurochem Res 20:491-496.

Stewart TL, Michel AD, Black MD, Humphrey PP (1996) Evidence that nitric oxide causes calcium-independent release of [3H] dopamine from rat striatum in vitro. J Neurochem 66:131-137.

Stys PK, Waxman SG, Ransom BR (1992) Ionic mechanisms of anoxic injury in mammalian CNS white matter: role of $\mathrm{Na}^{+}$channels and $\mathrm{Na}^{+}-\mathrm{Ca}^{2+}$ exchanger. J Neurosci 12:430-439.

Takeshita Y, Harata N, Akaike N (1996) Suppression of K+ conductance by metabotropic glutamate receptor in acutely dissociated large cholinergic neurons of rat caudate putamen. J Neurophysiol 76:1545-1558.

Vuillet J, Dimova R, Nieoullon A, Goff LK (1992) Ultrastructural relationships between choline acetyltransferase- and neuropeptide Y-containing neurons in the rat striatum. Neuroscience 46:351-360.

Wacholtz MC, Cragoe EJ Jr, Lipsky PE (1993) Delineation of the role of a Na ${ }^{+} / \mathrm{Ca}^{2+}$ exchanger in regulating intracellular $\mathrm{Ca}^{2+}$ in $\mathrm{T}$ cells. Cell Immunol 147:95-109.

Weiner DM, Levey AI, Brann MR (1990) Expression of muscarinic acetylcholine and dopamine receptor mRNAs in rat basal ganglia. Proc Natl Acad Sci USA 87:7050-7054

West AR, Galloway MP (1996) Intrastriatal infusion of $(+/-)-S$ nitroso- $N$-acetylpenicillamine releases vesicular dopamine via an ionotropic glutamate receptor-mediated mechanism: an in vivo microdialysis study in chloral hydrate-anesthetized rats. J Neurochem 66:1971-1980.

West AR, Galloway MP (1997) Endogenous nitric oxide facilitates striatal dopamine and glutamate efflux in vivo: role of ionotropic glutamate receptor-dependent mechanisms. Neuropharmacology 36:1571-1581.

West AR, Grace AA (2000) Striatal nitric oxide signaling regulates the neuronal activity of midbrain dopamine neurons in vivo. J Neurophysiol 83:1796-1808.

Zhu XZ, Luo LG (1992) Effect of nitroprusside (nitric oxide) on endogenous dopamine release from rat striatal slices. J Neurochem 59:932935 . 